\title{
Stabilizability of Discrete Chaotic Systems via Unified Impulsive Control
}

\author{
Honglei $\mathrm{Xu}^{*}$ Kok Lay Teo \\ Department of Mathematics and Statistics, Curtin University of Technology, \\ Perth, WA 6845, Australia
}

\begin{abstract}
This paper is concerned with the asymptotical stabilization problem of discrete chaotic systems by using a novel unified impulsive control scheme. Sufficient conditions for asymptotical stability of the impulsive controlled discrete systems are obtained by means of the Lyapunov stability theory and algebraic inequality techniques. Finally, numerical simulations on the Hénon and Ushio discrete chaotic systems are presented to illustrate the effectiveness and usefulness the unified impulsive control scheme.
\end{abstract}

Key words: Stability analysis, chaotic systems, impulsive control, nonlinear dynamics

PACS: 02.30.Yy, 05.45.Gg, 05.45.-a, 95.10.Fh

\section{Introduction}

Impulsive control has received a considerable interest in the past two decades. It is an effective control technique for achieving an excellent system performance, such as fast response time, low energy consumption, good robustness and resistance to disturbances. Many impulsive control strategies have been developed and applied to a wide range of applications, including chaos stabilization and synchronization, artificial neural networks, chemical process systems, drug administration in cancer chemotherapy, insulin injection, communication security and biological systems. For example, the Lyapunov functions method is employed to construct an impulsive control scheme in $[5,10]$

* Corresponding author. Tel.: +61 892664961; fax: +61 982663197.

Email address: H.Xu@curtin.edu.au (Honglei Xu). 
to asymptotically stabilize continuous chaotic systems. In [6], a rigorous analytical method for chaos stabilization is established for continuous chaotic systems. Also, the impulsive control theory is utilized in [7] to stabilize and synchronize continues chaotic systems under noise perturbations.

For communication security, an impulsive control-based scheme was adapted to synchronize two continuous chaotic systems (see [8] and the relevant references). In particular, the scheme that synchronizes the chaotic systems uses only small impulses. Numerical simulations exhibit an excellent performance in terms of synchronization and communication security. For a detailed discussion of impulsive control, impulsive differential equations and applications, see $[3,4,11,12]$ and relevant references cited therein.

The stabilization of chaotic oscillators is fundamentally important for nonlinear dynamics. It is also crucial for discrete chaotic systems. Some interesting results have been obtained (see, for example, $[9,13]$ and the references therein) by using the comparison principle and Lyapunov stability theory for impulsive discrete systems. Control and synchronization of discrete chaotic systems under the impulsive control-based scheme have been investigated in [14-16]. Note that these stability criteria are obtained under the assumption that the impulsive control gain matrix only appears linearly. As a consequence, the range of the applications of these results is limited. Therefore, it is desirable and challenging to develop a novel and effective impulsive control scheme for controlling discrete chaotic systems. In this paper, we shall propose a new unified impulsive control scheme whose impulsive gain matrix can be in the form of either linear functions or nonlinear functions. Besides, we derive several stability criteria for impulsive controlled discrete chaotic systems under the proposed unified impulsive control scheme. Numerical simulations can show that this new control scheme, which is constructed by using these novel stability criteria, can effectively asymptotically stabilize the discrete chaotic systems.

The rest of the paper is organized as follows. In Section II, a discrete chaotic system, a unified impulsive control scheme and some stability definitions are introduced. In Section III, new sufficient conditions for asymptotical stability for impulsive controlled discrete chaotic systems are established. In Section IV, two numerical examples are presented to demonstrate our main results. Concluding remarks are given in Section V.

\section{Unified impulsive control scheme}

Consider the following discrete chaotic system:

$$
x(m+1)=f(x(m))+u(x(m)),
$$


where $x=\left[x_{1}, x_{2}, \ldots, x_{n}\right]^{T} \in \mathbb{R}^{n}$ is the state vector, $m \in \mathbb{R}^{+}=\{0,1,2, \ldots\}$, $f: \mathbb{R}^{n} \rightarrow \mathbb{R}^{n}$ is a continuous nonlinear function and $u(x(m)) \in \mathbb{R}^{n}$ is an impulsive control input. Our main aim is to design a unified impulsive control input $u(x(m))$ such that system (1) under the control input is asymptotically stable. Let the sequence $\left\{n_{k}, u_{k}\left(x\left(n_{k}\right)\right)\right\}$ be the unified impulsive control input of system (1), i.e.,

$$
u(x(m))= \begin{cases}0, & m \neq n_{k} \\ \phi_{k}(x(m)), & m=n_{k},\end{cases}
$$

where $\phi_{k}: \mathbb{R}^{n} \rightarrow \mathbb{R}^{n}$ is a continuous linear or nonlinear function. Under this unified impulsive control scheme, the controlled discrete chaotic system can be described by the following impulsive difference equation:

$$
\begin{aligned}
x(m+1) & =f(x(m)), m \neq n_{k}, \\
\Delta x(m) & =\phi_{k}(x(m)), m=n_{k},
\end{aligned}
$$

where $\Delta x(m)=x(m+1)-x(m), \phi_{k} \in \mathbb{R}^{n}$ is the impulsive control input vector, and $f(0)=\phi_{k}(0)=0$. Denote by $x(m)$ the solution of system (2) with the initial condition $x(0)=x_{0}$. Let $n_{0}=0, n_{k}<n_{k+1}$ with $n_{k} \rightarrow \infty$ as $k \rightarrow \infty, k \in \mathbb{R}^{+}$.

Usually, the jumping impulses can not happen continuously, which means that the following assumption is satisfied.

Assumption 1 The impulsive control instants $\left\{n_{k}\right\}, n_{k} \in \mathbb{R}^{+}$, are such that

$$
n_{k}+2 \leq n_{k+1}, k \in \mathbb{R}^{+} .
$$

Definition 1 Let $x(m)=x(m ; 0, x(0))$ denote a solution of system (2). Then, system (2) is said to be stable if for any given $\varepsilon>0$, there exists a $\delta=\delta(\varepsilon)>0$ such that $\left\|x_{0}\right\| \leq \delta$ implies $\|x(m)\|<\varepsilon, \forall m \in \mathbb{R}^{+}$, for any $x(m)$.

Definition 2 System (2) is said to be asymptotically stable if it is stable and

$$
\lim _{m \rightarrow+\infty}\|x(m)\|=0 \text {. }
$$

\section{Criteria for stabilization}

In this section, we shall derive the sufficient conditions of asymptotical stability for the impulsive controlled discrete chaotic system (2) by employing the Lyapunov stability theory and algebraic inequality techniques. 
Theorem 1 Let Assumption 1 be satisfied, and suppose that

$$
\left\|x(m)+\phi_{k}(x(m))\right\| \leq l_{k}\|x(m)\|,
$$

where $\sum_{i=0}^{k} \ln l_{i}<0$, and

$$
\|f(x(m))\| \leq s\|x(m)\| .
$$

Then, system (2) is asymptotically stable if, for any $m \geq 3$, there exists a positive constant $\alpha>0$ such that

$$
\ln s+\frac{1}{m-1} \sum_{i=0}^{k} \ln l_{i} \leq-\alpha
$$

Proof. For $m \in\left(n_{k}, n_{k+1}\right], m \in \mathbb{R}^{+}$, we have

$$
\begin{aligned}
\|x(m)\| & =\|f(x(m-1))\| \\
& \leq s^{m-n_{k}-1}\left\|x\left(n_{k}+1\right)\right\| \\
& =s^{m-n_{k}-1}\left\|x\left(n_{k}\right)+\phi_{k}\left(x\left(n_{k}\right)\right)\right\| \\
& \leq s^{m-n_{k}-1} l_{k}\left\|x\left(n_{k}\right)\right\| .
\end{aligned}
$$

It is clear from (8) that

$$
\left\|x\left(n_{k+1}\right)\right\| \leq s^{n_{k+1}-n_{k}-1} l_{k}\left\|x\left(n_{k}\right)\right\|, k \in \mathbb{R}^{+} .
$$

By induction, we have

$$
\left\|x\left(n_{k}\right)\right\| \leq s^{n_{k}-k} \prod_{i=0}^{k-1} l_{i}\left\|x_{0}\right\|, \quad k \in \mathbb{R}^{+} .
$$

Thus, it follows from (8) and (10) that

$$
\begin{aligned}
\|x(m)\| & \leq s^{m-k-1} \prod_{i=0}^{k} l_{i}\left\|x_{0}\right\| \\
& =e^{(m-k-1) \ln s+\sum_{i=0}^{k} \ln l_{i}}\left\|x_{0}\right\| .
\end{aligned}
$$

It is clear from (3) that $n_{k} \geq 2 k+n_{0} \geq 2 k$, which indicates that $m \geq 2 k$. In addition, we have the following inequality:

$$
\frac{1}{m-1} \leq \frac{1}{m-k-1}, \quad \forall m \geq 3 .
$$

Then, it follows from (7) and (12) that 


$$
\begin{aligned}
& (m-k-1) \ln s+\sum_{i=0}^{k} \ln l_{i} \\
\leq & (m-k-1)\left(\ln s+\frac{1}{m-1} \sum_{i=0}^{k} \ln l_{i}\right) \\
\leq & \left(1-\frac{m}{2}\right) \alpha, \quad \forall m \geq 3 .
\end{aligned}
$$

Thus, by (11) and (13), we obtain

$$
\|x(m)\| \leq e^{\left(1-\frac{m}{2}\right) \alpha}\left\|x_{0}\right\|, \forall m \geq 3 .
$$

Now, by Definition 1, we see that the impulsive controlled discrete chaotic system (2) is stable. In addition, it follows from (14) that

$$
\lim _{m \rightarrow+\infty}\|x(m)\|=0
$$

Therefore, the impulsive controlled discrete system (2) is asymptotically stable. The proof is complete.

In the following, we show that Theorem 1 can be specialized to a special case, where all nonlinear functions $\phi_{k}(x)$ are identical at the impulsive control instants $n_{k}$, i.e., $\phi_{k}(x)=\phi(x), \forall k \in \mathbb{R}^{+}$. We assume that the following assumption is satisfied.

Assumption 2 The impulsive control instants $\left\{n_{k}\right\}, n_{k} \in \mathbb{R}^{+}$, are such that

$$
n_{k+1} \leq n_{k}+4, k \in \mathbb{R}^{+} .
$$

In the following, Theorem 1 is specialized to the case mentioned above.

Theorem 2 Let Assumptions 1-2 be satisfied. Suppose that

$$
\|x(m)+\phi(x(m))\| \leq l\|x(m)\|
$$

where $0<l<e$, and

$$
\|f(x(m))\| \leq s\|x(m)\|
$$

Then, system (2) is asymptotically stable if

$$
\ln s+\frac{1}{3} \ln l<0 .
$$

Proof. Suppose that $m \in\left(n_{k}, n_{k+1}\right]$ for any $m \in \mathbb{R}^{+}$. Then it follows from (11) appearing in the proof of Theorem 1 that

$$
\|x(m)\| \leq e^{(m-k-1) \ln s+(k+1) \ln l}\left\|x_{0}\right\| .
$$


By Lemma 2 and noting that $0<l<e$, we obtain

$$
\begin{aligned}
& (m-k-1) \ln s+(k+1) \ln l \\
= & (m-k-1)\left(\ln s+\frac{k+1}{m-k-1} \ln l\right) \\
\leq & (m-k-1)\left(\ln s+\frac{1}{3} \ln l\right) .
\end{aligned}
$$

Substituting (21) into (20) yields

$$
\|x(m)\| \leq e^{\left(\frac{m}{2}-1\right)\left(\ln s+\frac{1}{3} \ln l\right)}\left\|x_{0}\right\|, m \in \mathbb{R}^{+} .
$$

Then, it follows from (19) and (22) that there exists a $\sigma=-\left(\ln s+\frac{1}{3} \ln l\right)>0$ such that the following inequality holds,

$$
\|x(m)\| \leq e^{\left(1-\frac{m}{2}\right) \sigma}\left\|x_{0}\right\|, \quad \forall m \in \mathbb{R}^{+},
$$

which shows that the impulsive controlled discrete chaotic system (2) is stable. In addition, by (23), we have $\lim _{m \rightarrow+\infty}\|x(m)\|=0$. Therefore, the impulsive controlled discrete chaotic system (2) is asymptotically stable. The proof is complete.

\section{EXPERIMENTAL RESULTS}

In this section, we present two examples to illustrate the effectiveness of our unified impulsive control scheme.

Example 1: Consider the 3-dimensional generalization of the Hénon map [1]:

$$
\left\{\begin{array}{l}
x_{1}(m+1)=1-a x_{1}^{2}(m)+x_{2}(m), \\
x_{2}(m+1)=b x_{1}(m)+x_{3}(m), \\
x_{3}(m+1)=-b x_{1}(m) .
\end{array}\right.
$$

$\forall m \in \mathbb{R}^{+}, x_{0}=[-1.2,0,0.3]^{T}$. When $a=1.07, b=0.3$, the discrete system (24) is chaotic. The chaotic behaviors of system (24) are presented in Figure 1-Figure 3. The corresponding phase portrait is depicted in Figure 4. It is clear that $[-1.54,0,0.46]^{T}$ is one of the equilibrium points of system (24). Our aim is to design an appropriate impulsive controller to asymptomatically stabilize system $(24)$ to the fixed point $[-1.54,0,0.46]^{T}$. First, let us shift this equilibrium point to the trivial equilibrium point. Let $y_{1}(t)=x_{1}(t)+1.54$, 
$y_{2}(t)=x_{2}(t), y_{3}(t)=x_{3}(t)-0.46$. Then, the Hénon chaotic system (24) can be rewritten as:

$$
\left\{\begin{array}{l}
y_{1}(m+1)=-1.07 y_{1}^{2}(m)+3.3 y_{1}(m)+y_{2}(m) \\
y_{2}(m+1)=0.3 y_{1}(m)+y_{3}(m) \\
y_{3}(m+1)=-0.3 y_{1}(m)
\end{array}\right.
$$

By (5), we design a nonlinear impulsive controller as $\left\{n_{k}, u_{k}(y)=\phi_{k}(y)\right\}$, where

$$
\begin{gathered}
u_{k}(y(m))=\phi_{k}(y) \\
=\left(-e^{-\frac{3(m-1)}{(k+1)}} \sqrt{\left|y_{2} y_{3}\right|}-y_{1},-e^{-\frac{3(m-1)}{(k+1)}} \sqrt{\left|y_{1} y_{3}\right|}-y_{2},\right. \\
\left.-e^{-\frac{3(m-1)}{(k+1)}} \sqrt{\left|y_{1} y_{2}\right|}-y_{3}\right)^{T} .
\end{gathered}
$$

Let $n_{k}=8 m, m \in \mathbb{R}^{+}$and choose $s=\max \left\{1.1449 \bar{y}_{1}^{2}+7.062\left|\bar{y}_{2}\right|+2.14\left|\bar{y}_{3}\right|+\right.$ $0.48,14.19\}$, where $\bar{y}_{i}=\sup _{k \in \mathbb{R}^{+}} \|\left\{y_{i}(k) \|\right\}, i=1,2,3$, which represents the maximum Euclidean norm of $f(x(m))$. Then, through numerical simulation in the MATLAB environment, we find $\bar{y}_{1}=2.9706, \bar{y}_{2}=0.8461$ and $\bar{y}_{3}=0.8912$. Thus, we obtain $s=18.1655, l_{k}=e^{-\frac{3(m-1)}{(k+1)}}$.

In addition, we have the following inequality:

$$
\ln s+\frac{1}{m-1} \sum_{i=0}^{k} \ln l_{i}=-0.0841<0 .
$$

Therefore, by Theorem 1, we see that the Hénon chaotic system (26) is asymptotically stable under the nonlinear impulsive controller $\left\{n_{k}, u_{k}(x)=\phi_{k}(x)\right\}$. Figure 5-Figure 7 depict the state trajectories of the discrete Hénon chaotic system (24) under the nonlinear impulsive control. Figure 8 depicts the corresponding phase portrait of the controlled discrete Hénon chaotic system (25).

Example 2: Consider the following Ushio discrete chaotic system [2]:

$$
\left\{\begin{array}{l}
x_{1}(m+1)=1.9 x_{1}(m)-x_{1}^{3}(m)+x_{2}(m), \\
x_{2}(m+1)=0.5 x_{1}(m)
\end{array}\right.
$$

The initial value is given as $x(0)=[0.6,-0.3]^{T}$. State trajectories of $x_{1}(m)$ and $x_{2}(m)$ of the Ushio discrete chaotic system (26) are depicted in Figure 9 and Figure 10. Phase portrait of the discrete chaotic system (26) is depicted in Figure 11. According to (17), we devise a simple linear impulsive controller as $\left\{n_{k}, u_{k}(x)=\phi_{k}(x)\right\}$, where

$$
u_{k}(x(m))=\phi_{k}(x)=\left(e^{-9}-1\right) x .
$$




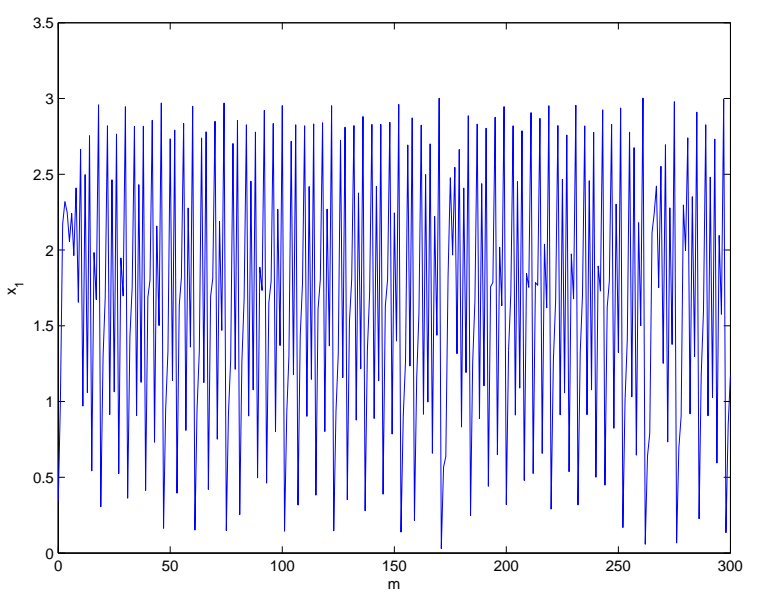

Fig. 1. State trajectory of $x_{1}(m)$ of the Hénon discrete chaotic system (25).

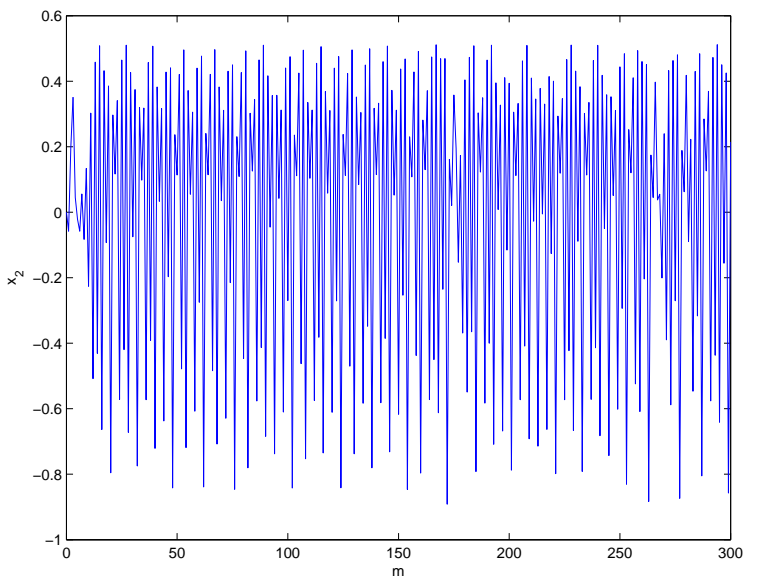

Fig. 2. State trajectory of $x_{2}(m)$ of the Hénon discrete chaotic system (25).

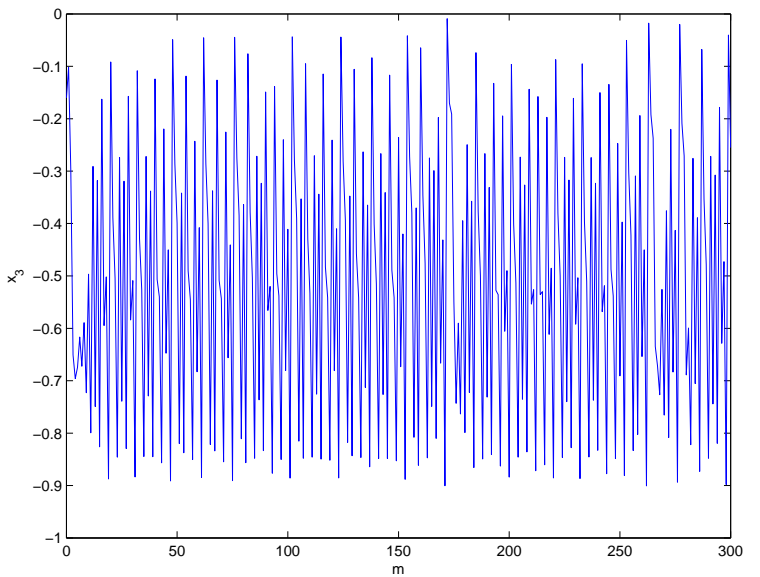

Fig. 3. State trajectory of $x_{3}(m)$ of the Hénon discrete chaotic system (25). 


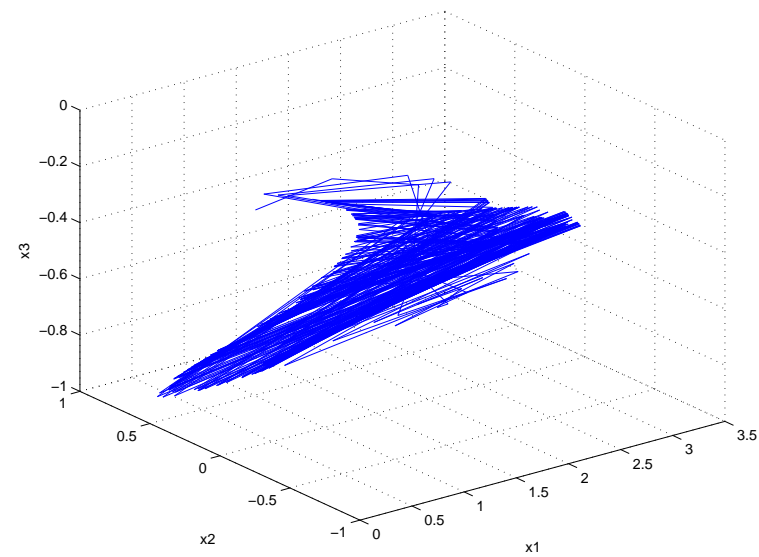

Fig. 4. Phase portrait of the Hénon discrete chaotic system (25).

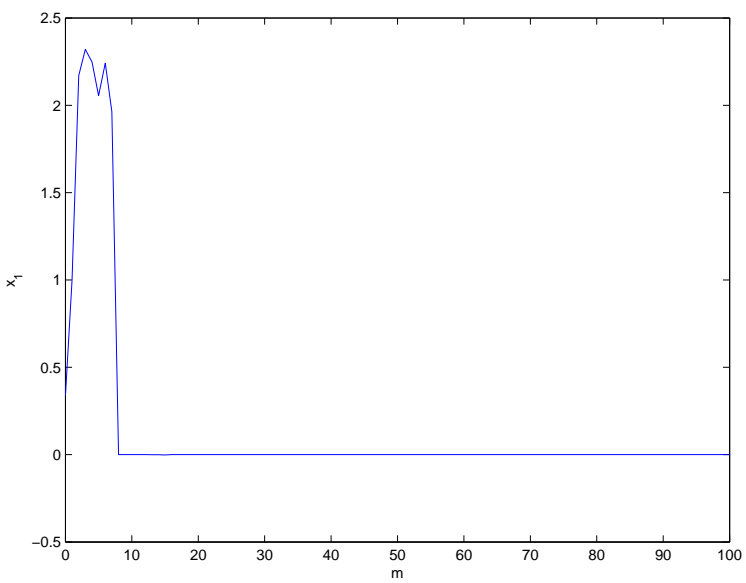

Fig. 5. State trajectory of $x_{1}(m)$ of the Hénon discrete chaotic system (25) under the nonlinear impulsive control.

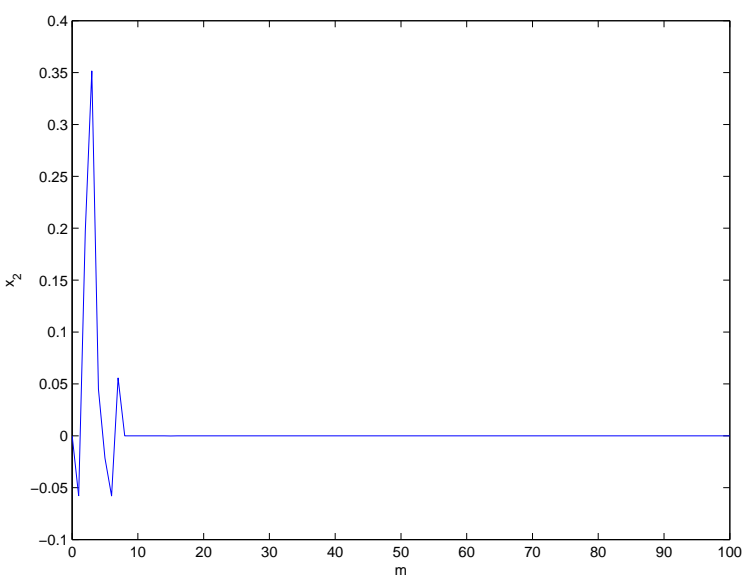

Fig. 6. State trajectory of $x_{2}(m)$ of the Hénon discrete chaotic system (25) under the nonlinear impulsive control. 


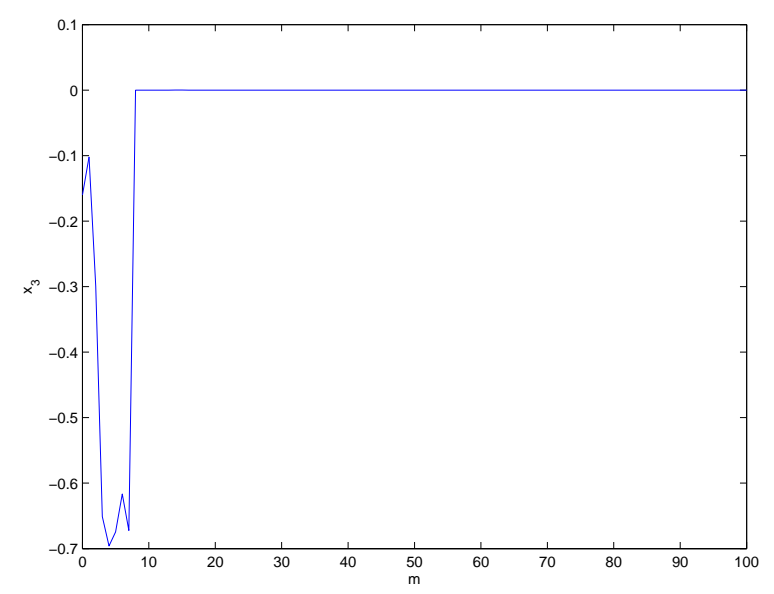

Fig. 7. State trajectory of $x_{3}(m)$ of the Hénon discrete chaotic system (25) under the nonlinear impulsive control.

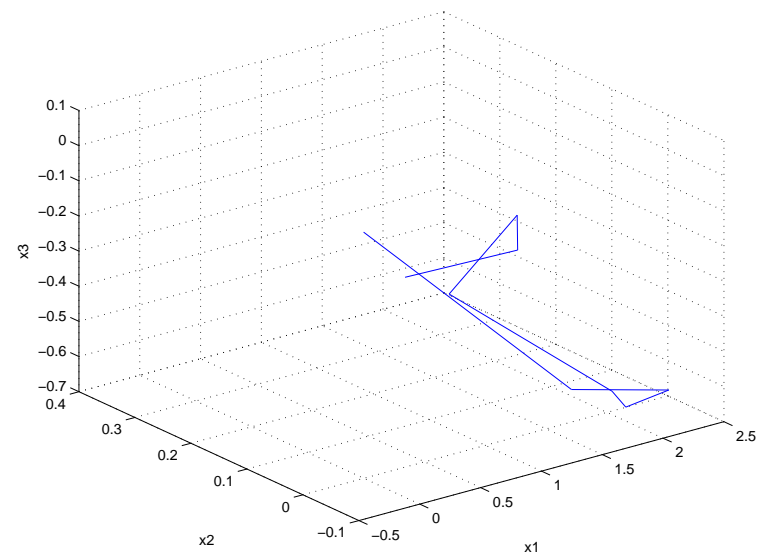

Fig. 8. Phase portrait of the Hénon discrete chaotic system (25) under the nonlinear impulsive control.

Let $n_{k}=3 m, m \in \mathbb{R}^{+}$and choose $s=\max \left\{6.01+2 \bar{x}_{1}^{4}, 3.9\right\}$, where $\bar{x}_{1}=$ $\sup _{m \in \mathbb{R}^{+}}\left\{x_{1}(m)\right\}=1.6577$. Then, we find $s=15.1121, l=e^{-9}$. It is easy to verify that

$$
\ln s+\frac{1}{3} \ln l=\ln s+\frac{1}{3} \ln e^{-9} \approx-0.2845<0,
$$

which satisfies (7).

By Theorem 2, we conclude that the discrete chaotic system (26) is asymptotically stable under the linear impulsive controller $\left\{n_{k}, u_{k}(x)=\phi_{k}(y)\right\}$. The state trajectories of $y_{1}(m)$ and $y_{2}(m)$ of the controlled Ushio chaotic systems and their phase portrait are depicted in Figure 12-Figure 14, respectively. Numerical Simulations show that our designed controllers are highly effective for stabilizing Ushio discrete chaotic systems. 


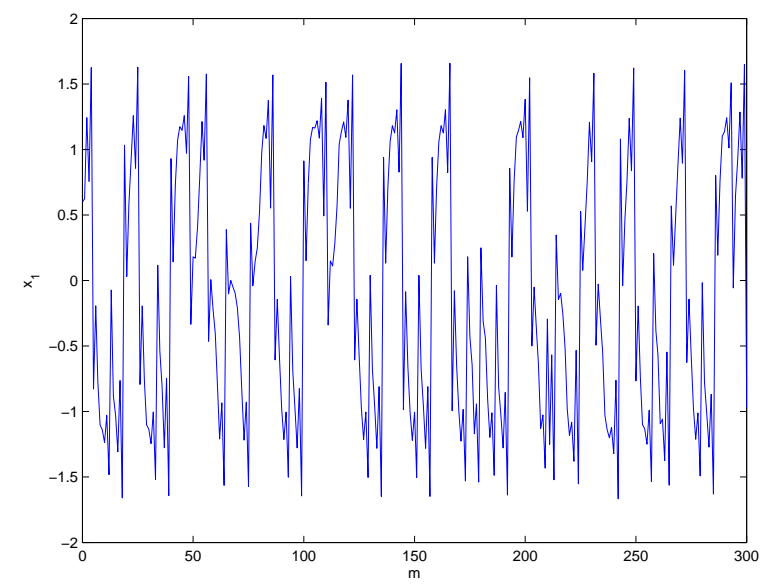

Fig. 9. State trajectory of $x_{1}(m)$ of the Ushio discrete chaotic system (26).

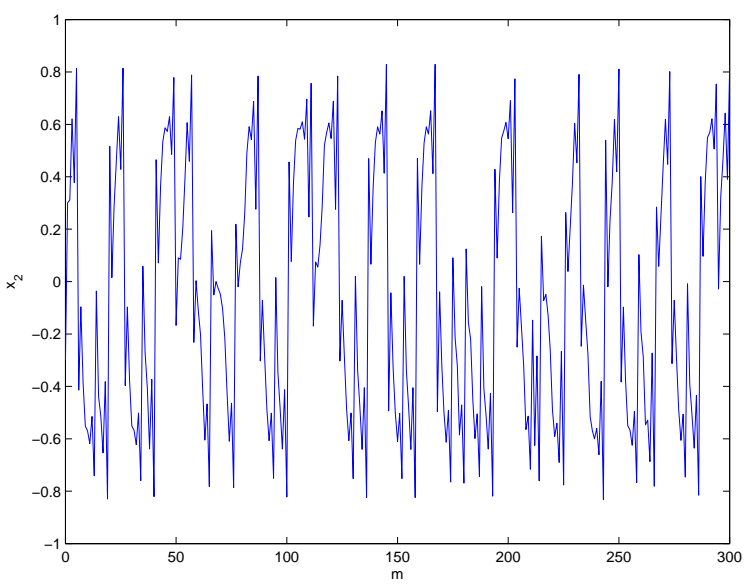

Fig. 10. State trajectory of $x_{2}(m)$ of the Ushio discrete chaotic system (26).

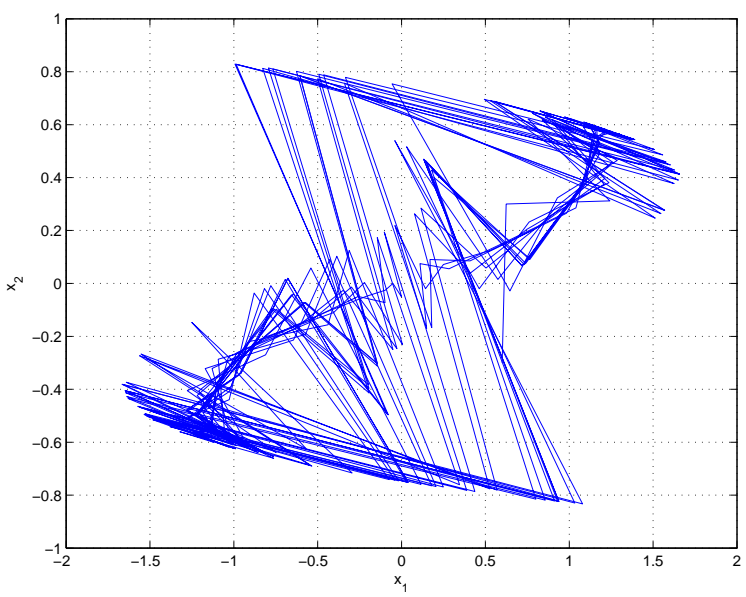

Fig. 11. Phase portrait of the Ushio discrete chaotic system (26). 


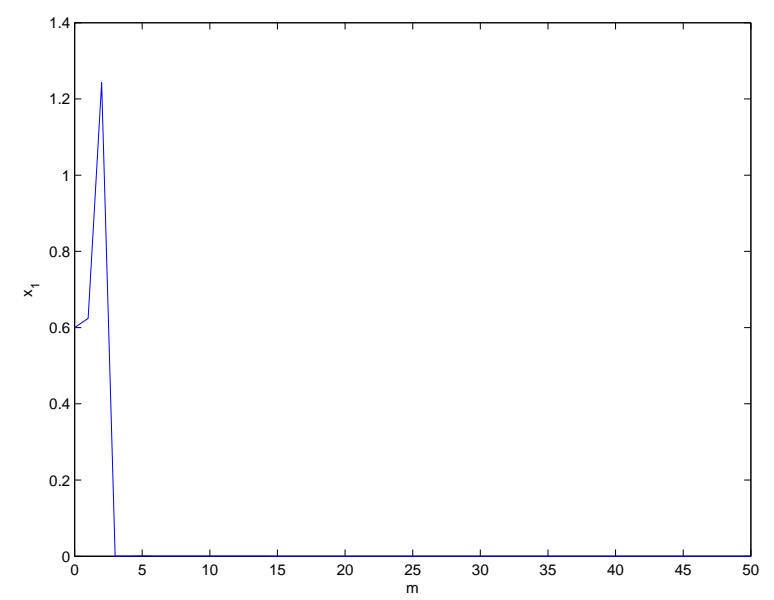

Fig. 12. State trajectory of $x_{1}(m)$ of the Ushio discrete chaotic system (26) under the nonlinear impulsive control.

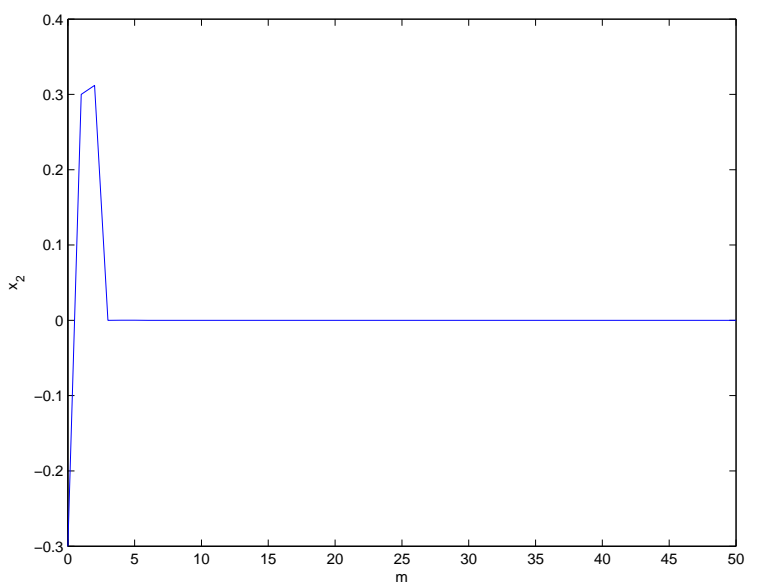

Fig. 13. State trajectory of $x_{2}(m)$ of the Ushio discrete chaotic system (26) under the nonlinear impulsive control.

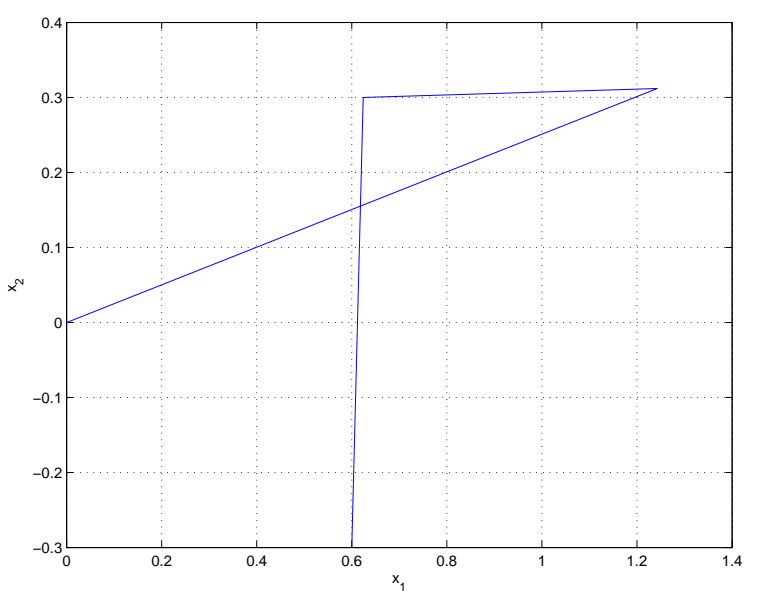

Fig. 14. Phase portrait of the Ushio discrete chaotic system (26) under the nonlinear impulsive control. 


\section{CONCLUSION}

In this paper, we proposed a novel design scheme (unified impulsive stabilizing control scheme) for discrete chaotic systems. Sufficient conditions for asymptotical stability of the impulsive controlled discrete chaotic systems were derived by employing the Lyapunov stability theory. From the numerical studies, we see that the unified impulsive control scheme is highly effective.

\section{ACKNOWLEDGMENT}

This work was partially supported by the National Natural Science Foundation of China under Grant 60704003, an Australian Research Council Discovery Project, the Natural Science Foundation of Guizhou Province under Grant [2008]2252, and the Talents Foundation of Guizhou University under Grant 2007043.

\section{References}

[1] L. H. Donald, Z. Frank, An exploration of the Henon quadratic map, Physica D (14) (1985) 305-326.

[2] T. Ushio, Chaotic synchronization and controlling chaos based on contraction mappings, Phys. Lett. A (198) (1995) 14-22.

[3] V. Lakshmikantham, D. D. Bainov, P. S. Sineonov, Theory of Implusive Differentical Equations, World Scientific press, Singapore, 1989.

[4] T. Yang, Impulsive Control Theory, Springer, Berlin, 2001.

[5] X. Liu, K. L. Teo, Impulsive control of chaotic system, Int. J. of Bifurcation Chaos (12) (2002) 1181-1190.

[6] W. Xie and C. Wen and Z. Li, Impulsive control for the stabilization and synchronization of Lorenz systems, Phys. Lett. A (275) (2000) 67-72.

[7] C. Li, L. Chen, K. Aihara, Impulsive control of stochastic systems with applications in chaos control, chaos synchronization, and neural networks, Chaos (18) (2008) 023132.

[8] A. Khadra, X. Liu, X. Shen, Impulsively synchronizing chaotic systems with delay and applications to secure communication, Automatica (41) (2005) 14911502 .

[9] B. Liu, X. Liu, Robust stability of uncertain discrete impulsive systems, IEEE Trans. Circuit Syst. II,Exp. Briefs (54) (2007) 455-459. 
[10] T. Yang, Li. Yang, C. Yang, Impulsive control of Lorenz system, Physica D 110 (1997) 18-24.

[11] Y. J. Sun, Controlling chaotic Lu systems using impulsive control, Phys. Lett. A 342 (2005) 256-262.

[12] L. Wang, W. Xu, Y. Li, Impulsive control of a class of vibro-impact systems, Phys. Lett. A 372 (2008) 5309-5313.

[13] B. Liu and X. Z. Liu, Uniform stability of discrete impulsive systems, Int. J. Sys. Sci. (39) (2008) 181-192.

[14] Y. Zheng, Y. Nian, Z. Liu, Impulsive control for the stabilization of discrete chaotic system, Chin. Phys. Lett. (19) (2002) 1251-253.

[15] Y. Zheng, Y. Nian, Z. Liu, Impulsive synchronization of discrete chaotic systems, Chin. Phys. Lett. (20) (2003) 199-201.

[16] X. Zhang, X. Liao, C. Li, Impulsive control, complete and lag synchronization of unified chaotic system with continuous periodic switch, Chaos Solitons Fractals (26) (2005) 845-54. 